\title{
Metaphyseal tibial level (MTL) screws: a modified percutaneous technique for lateral plateau depression fractures
}

\author{
Frédéric Vauclair ${ }^{1,2} \cdot$ Mahmoud Almasri $^{2} \cdot$ Nicolas Gallusser $^{1} \cdot$ Hans Van Lanker ${ }^{2}$. \\ Rudy Reindl ${ }^{2}$
}

Received: 28 March 2015/ Accepted: 9 April 2015/Published online: 9 May 2015

(C) Springer-Verlag France 2015

\begin{abstract}
Introduction Lateral tibial plateau fractures are more frequent than medial fractures, and those with articular depression are particularly challenging because of high displacement risk. To prevent secondary subsidence, the gold standard is raft screws with a periarticular or antiglide plate. Graft is used to fill the metaphyseal defect created by reduction in the depressed fragment. We present a case of Schatzker II fracture managed in a complete percutaneous fashion, with a new combined technique of raft screws and interference screw used as a support.

Case report A 51-year-old female sustained a Schatzker II tibial plateau fracture. Based on pre-operative CT, direction of reduction force to apply was drawn on coronal and sagittal cuts.

Operative technique Under fluoroscopic control, the split component of the fracture was reduced. The cortical window was then drilled in the lateral cortex, and a $\mathrm{K}$ wire advanced under the depressed fragment under fluoroscopic guidance. After fragment reduction with a bone impacter, internal fixation was completed by percutaneous introduction of two subchondral cortical screws. A bioabsorbable interference screw was then introduced in the impacter
\end{abstract}

Nicolas Gallusser

nicolas.gallusser@chuv.ch

Frédéric Vauclair

frederic.vauclair@chuv.ch

1 Département de l'appareil locomoteur, Service d'orthopédie et traumatologie, Centre Hospitalier Universitaire Vaudois, Rue du Bugnon 46, BH 10-238, 1011 Lausanne, Switzerland

2 Montreal General Hospital, Division of Orthopaedic Surgery, Orthopaedic Trauma, McGill University Health Centre, 1650 Cedar Avenue, Montreal, QC H3G 1A4, Canada tunnel to support impacted bone under the reduced articular surface. Finally, a cortical screw was introduced, from anterior to posterior to prevent screw cut-out.

Conclusion The combination of subchondral screws in a jail technique with a bioabsorbable interference screw that we named metaphyseal tibia level (MTL) screw technique is, to our knowledge, not described. The MTL screw promises to be a true percutaneous reduction and fixation technique for Schatzker II and III fractures in patients with reasonable bone quality.

Keywords Percutaneous $\cdot$ Modified $\cdot$ Technique $\cdot$ Tibia Plateau · Fracture

\section{Introduction}

Tibial plateau fractures represent $1 \%$ of all fractures $[1,2]$ and 5-8 \% of lower limb fractures [3]. The incidence shows a bimodal distribution, with a first peak encountered in the second to fifth decade - males with high-energy trauma such as motor vehicle accidents-and a second in the fifth to seventh decade - most commonly females, during low-energy trauma as in osteoporotic fracture-[4]. In osteoporotic elderly patients, this fracture represents as much as $10 \%$ of all fractures [5].

Several fracture classifications exist, and the most widely used is the Schatzker classification. This system can be subdivided into three subgroups: types I-III are mostly of low- to middle-energy trauma, and they involve lateral plateau. Type IV affects the medial plateau. Types V-VI involve both the medial and the lateral plateau and are secondary to high-energy trauma. Lateral plateau fractures are more frequent than medial. Schatzker type II (also known as split-depression type) is the most frequent and is responsible for $25-33 \%$ of plateau fractures [6] and 50-84\% of lateral plateau fractures [7]. 
Current concepts in fracture management advocate the use of CT scan to better define articular congruity and fracture displacement. Surgical treatment is recommended for an articular step greater than 2-3 mm of articular step, plateau widening of more than $5 \mathrm{~mm}$ or valgus axis deviation $>5^{\circ}$. However, these guidelines based on displacement are well accepted, but based on opinion only [4].

The goal is to restore the joint congruence, mechanical axis and range of motion [8]. For Schatzker II and III (split-depression and depression), the gold standard is open reduction and internal fixation with a plate and screws. Some surgeons prefer the arthroscopic-assisted technique-particularly in type III, pure depression fracture-to benefit from an under-vision control of the reduction, without the need for arthrotomy. Others are confident in fluoroscopic views and address the fracture in a percutaneous approach.

Regarding fixation technique, there are a lot of biomechanical studies comparing lag screws, different type of plates (locking or non-locking) and raft screws [7]. The challenging part of these fractures is to prevent secondary subsidence of the depression. The golden standard is raft screws with a periarticular or anti-glide plate; more screws of smaller diameter, typically $3.5 \mathrm{~mm}$, that are parallel and close to subchondral bone achieve better stability $[9,10]$. Autologous bone graft is then used to fill the metaphyseal defect created by reduction in the depressed fragment. Some surgeons prefer allograft [11] to avoid morbidity related to graft-site harvesting (i.e. iliac crest) [12], but the actual trend is the use of calcium phosphate cement, which has been shown to have a higher resistance to load stress $[13,14]$. Hydroxyapatite calcium carbonate synthetic bone graft is also an alternative [15].
We present a case in which we chose to manage a Schatzker type II fracture (split-depression) in a complete percutaneous fashion, under fluoroscopic control and with a new combined technique of raft screws and bioabsorbable screw (the one designed for anterior cruciate ligament graft fixation).

\section{Informed consent}

The patient was informed that data concerning her case would be submitted for publication. Her consent was obtained.

\section{Case report}

The patient is a 51-year-old female who sustained a lowvelocity motorcycle accident. After initial trauma work-up, our orthopaedic trauma team saw her for pain in the right knee. Clinical examination showed swelling of the knee, pain localized on the lateral proximal tibia without any laxity. Neurovascular status was normal. A Schatzker type II tibial plateau fracture (Ao 41 B3) was visible on standard $\mathrm{X}$-ray, with some extension of the line through the tibial spines. CT scan (Fig. 1) better defined the fracture pattern and confirmed the extension. After signed consent was obtained, surgery was performed on the patient.

\section{Operative technique description}

Based on pre-operative CT, we planned to do percutaneous reduction in the articular depression through a lateral

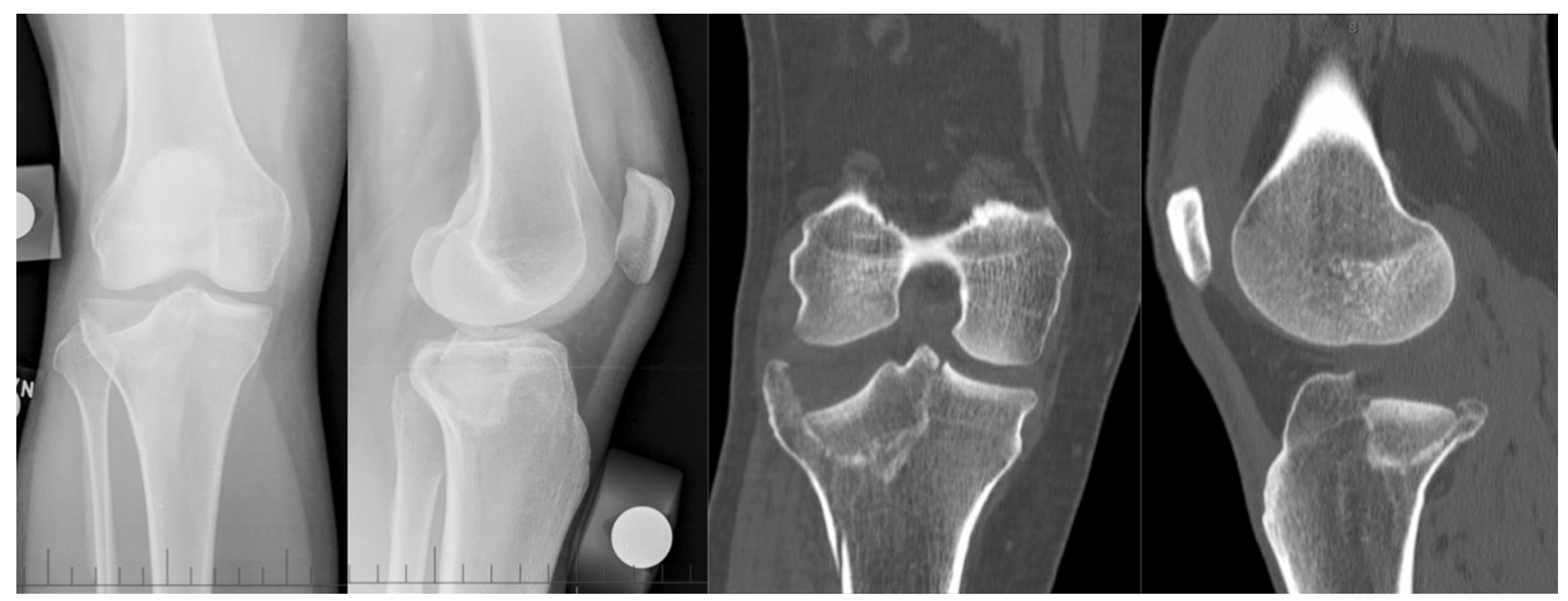

Fig. 1 (Left) AP and lateral X-ray of the knee showing a depression fracture of the lateral tibia plateau. (Right) Coronal and sagittal CT scan better defining the fracture pattern, confirming the depression and showing a split as well as some extension of the line through the tibial spines (Schatzker type II tibial plateau fracture) 


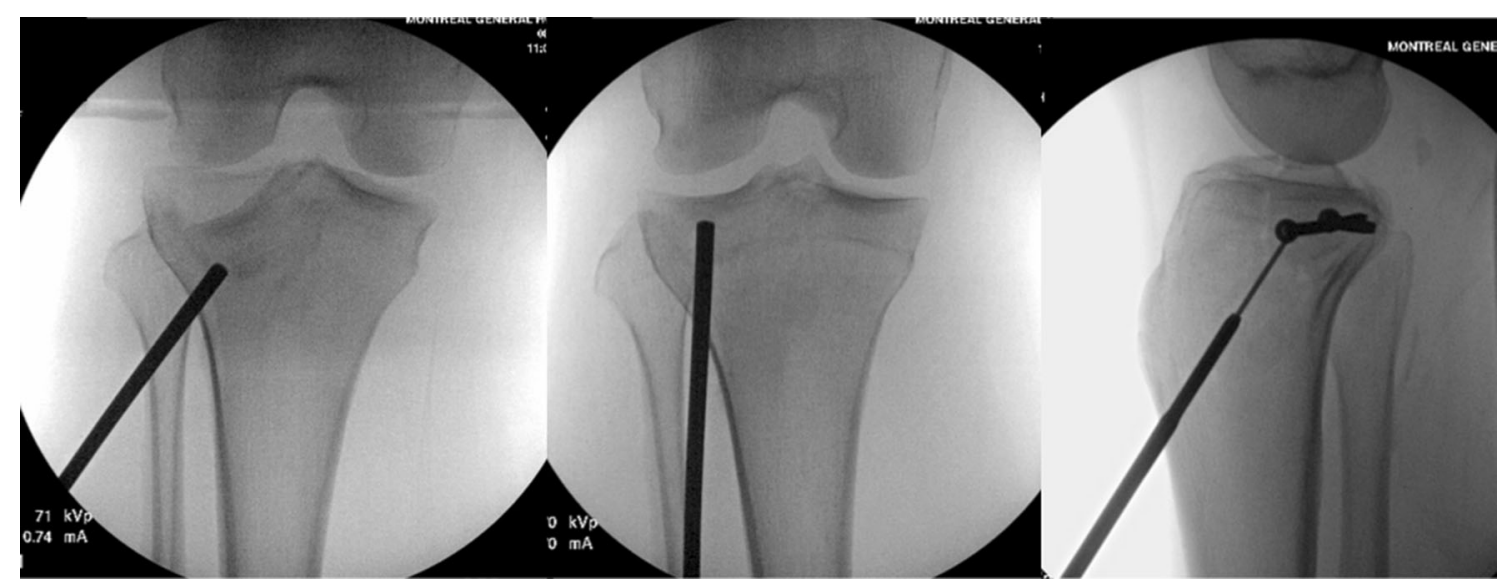

Fig. 2 (Left) Fluoroscopic images of fragment reduction using a bone impacter. (Right) After percutaneous introduction of two subchondral $3.5-\mathrm{mm}$ cortical screws under the reduced articular surface (raft screws), a bioabsorbable interference screw (Smith and Nephew

cortical window. Direction of reduction force to apply was drawn on both coronal and sagittal cuts.

Patient was supine on a Jackson table under regional anaesthesia. She received $2 \mathrm{~g}$ of intravenous cefazolin for infection prophylaxis. Non-sterile tourniquet was applied. The right knee was prepared, draped and flexed to $30^{\circ}$ using a radiolucent triangle. Under fluoroscopic control, the split component of the fracture was reduced with a Weber clamp. The cortical window was then drilled in the lateral cortex, and a $\mathrm{K}$ wire advanced under the depressed fragment with the direction chosen on pre-operative CT (anterior to posterior), under both AP and lateral fluoroscopic guidance. After planned axis was obtained, we proceed to fragment reduction using a bone impacter. Internal fixation was then completed by percutaneous introduction of two subchondral $3.5-\mathrm{mm}$ cortical screws (raft lateral to medial, under the reduced articular surface). A bioabsorbable screw (Smith and Nephew $8 \mathrm{~mm} \times 35 \mathrm{~mm}$ BIORCI screw) was then introduced through the bone impacter tunnel to strongly support impacted bone under the reduced articular surface, with the same vector as that of the reduction (according to pre-operative planning) (Fig. 2). Finally, an additional 3.5-mm cortical screw was introduced, from anterior to posterior to prevent screw cutout (jail technique) [3].

Final fluoroscopic shots were taken (Fig. 3) to confirm the anatomic reduction in the fracture. Skin was closed with 3.0 nylon and dry dressing applied.

Patient was allowed to have immediate range of motion as tolerated but without weight bearing for the first 6 weeks, progressive weight bearing for the next 6 weeks. She received 6 weeks of subcutaneous low molecular weight heparin.

Post-operative course was uneventful. At 4-month follow-up, the patient had recovered full range of motion and
$8 \mathrm{~mm} \times 35 \mathrm{~mm}$ BIORCI screw) was then introduced through the bone impacter tunnel to strongly support impacted bone under the reduced articular surface, with the same vector as reduction's one

the wounds were barely visible. At last follow-up, the patient was asymptomatic. One-year X-ray did not show any evidence of displacement or subsidence (Fig. 4).

\section{Discussion}

Lateral tibial plateau fractures are more frequent than medial fractures. Those with pure articular depression (Schatzker type III, Ao 41 B2) or split-depression (Schatzker type II, Ao 41 B3) are particularly challenging because of high secondary displacement risk. Quality and maintenance of anatomic reduction is mandatory to prevent, as much as possible, knee osteoarthritis, which is the major medium-term and long-term complication, with $12-63 \%$ of patients demonstrating radiological signs of $\mathrm{OA}$ in the medium-term [2].

As described above, numerous types of grafting (bone auto- and allograft, calcium phosphate cement, hydroxyapatite synthetic bone) have been used for metaphyseal bone defect filling and articular fragment subsidence prevention.

Regarding fixation, screw positioning is of great importance, and technique with raft of screws is the gold standard.

The interference screw technique (bioabsorbable screw in the tunnel) was first described by Lubowitz et al. [16] for arthroscopic reduction and fixation of depression fracture without any other fixation. However, a recent biomechanical study in porcine bone has shown that a construct with this screw only achieves a lower strength than with two subchondral screws [17]. Thus, we think that this technique should not be used alone.

We have chosen to use the jail technique, first described by Weinmann et al. [3] in a biomechanical study, which consists in raft screws with additional anterior-to-posterior 
Fig. 3 Final AP and lateral images showing the two raft screws and an additional 3.5$\mathrm{mm}$ cortical anterior-toposterior screw (to prevent screw cut-out) with confirmation of the anatomic reduction of the fracture

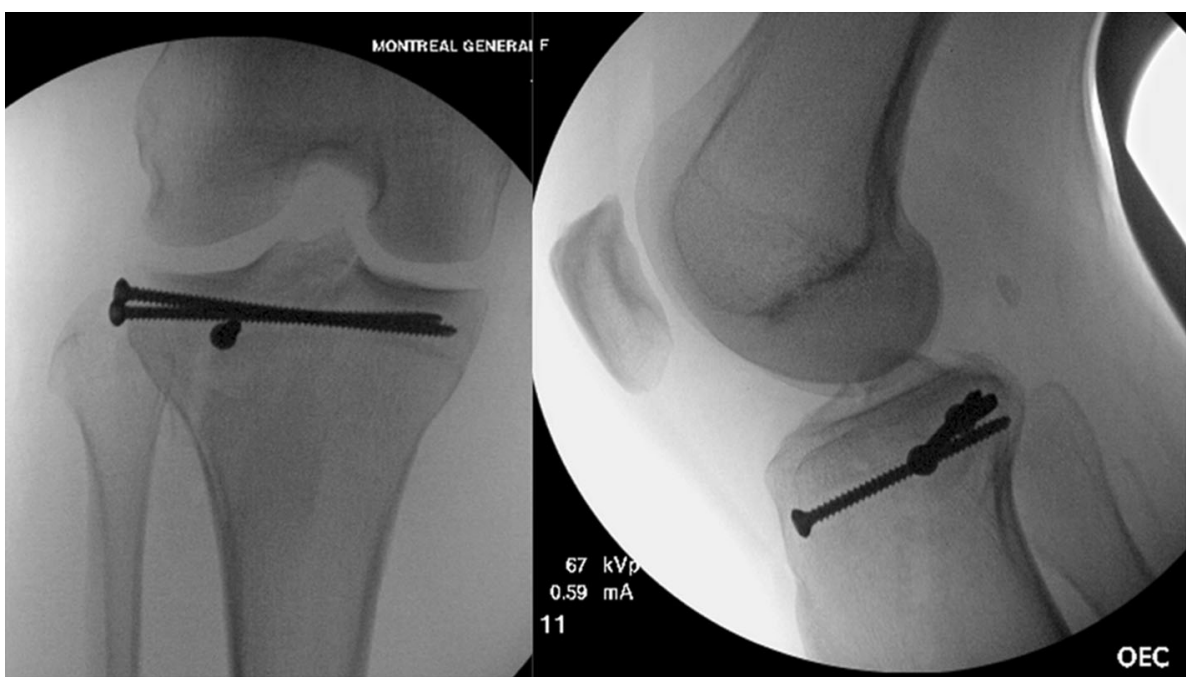

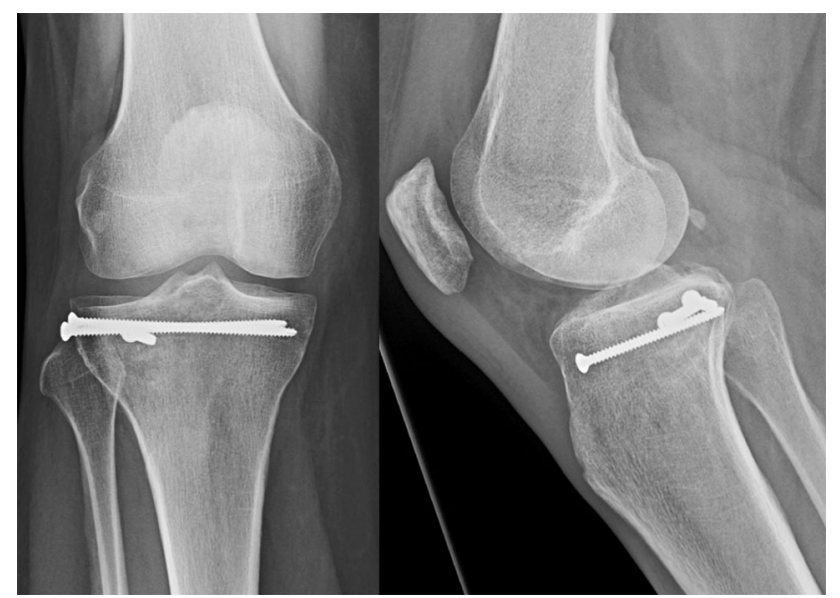

Fig. 4 At 12-month follow-up, the fracture is healed and X-ray did not show any displacement or subsidence

screw (to prevent screw cut-out), and decided to combine this technique with a bioabsorbable screw as a graft. The bioabsorbable screw has many advantages. First of all, it brings a strong support to the impacted bone under the reduced articular surface (because of its inherent stability in metaphyseal bone, the same way it does for ACL reconstruction), and this support has the same force vector as the reduction vector. It fills the bone tunnel, and therefore, no further grafting technique or cement is needed. Finally, bioabsorbable screws are easy to use and have no learning curve.

The combination of subchondral screws in a jail technique with a bioabsorbable screw that we named metaphyseal tibia level (MTL) screw technique is, to our knowledge, not described in the literature. Even if it is only a modification of other techniques, it is based on biomechanical and clinical published data, which support its efficiency.
This modified technique should be a strong and safe technique to address depression or split-depression fractures of lateral tibia plateau in patient with good bone quality. Of course, the fracture has to be not too comminuted to be reduced through a single bone tunnel, and depending on the fracture pattern, a periarticular plate might be needed to complete the construct. This combined technique has the advantage of a true percutaneous technique minimizing infection risk which is $2-11 \%$ in open technique [8]. It might be the counterpart to the technique of balloon reduction with calcium phosphate cement [18] which is, from our point of view, more suitable for depression fracture with big voids in osteoporotic elderly patients. In addition, balloon osteoplasty has a high rate of technical complications (up to $65 \%$, mostly related to the learning curve). For orthopaedic trauma surgeons, this technique is feasible without arthroscopic skills.

Regardless of fixations technique, patients should still be kept non-weight bearing until bone union is acquired. In fact, Karunakar et al. [9] in a biomechanical study on cadaver have pointed out persistent weakness regardless of construct.

Finally, we strongly recommend the use of pre-operative MRI to rule out associated meniscal injury (found in 50-90 \% in some series) [1, 10, 19]. Ringus et al. [20] have shown a fourfold increase in risk of lateral meniscus tear in patients younger than 48 years and an almost eightfold increase in risk of patient with $>10 \mathrm{~mm}$ of depression.

In conclusion, the MTL screw approach promises to be a true percutaneous reduction and fixation technique for Schatzker II and III fractures in patients with reasonable bone quality.

Acknowledgments Dr Vauclair would like to thank the Swiss Society of Orthopaedics and Traumatology and the SICPA foundation 
for their financial support which render his trauma fellowship at the Montreal General Hospital (McGill University) possible.

Conflict of interest None.

\section{References}

1. Chan YS (2011) Arthroscopy-assisted surgery for tibial plateau fractures. Chang Gung Med J 34(3):239-247

2. Siegler J, Galissier B, Marcheix PS, Charissoux JL, Mabit C, Arnaud JP (2011) Percutaneous fixation of tibial plateau fractures under arthroscopy: a medium term perspective. Orthop Traumatol Surg Res 97(1):44-50. doi:10.1016/j.otsr.2010.08.005

3. Weimann A, Heinkele T, Herbort M, Schliemann B, Petersen W, Raschke MJ (2013) Minimally invasive reconstruction of lateral tibial plateau fractures using the jail technique: a biomechanical study. BMC Musculoskelet Disord 14(1):120. doi:10.1186/14712474-14-120

4. Bucholz RW, Heckman JD, Court-Brown CM, Tornetta P III (2009) Rockwood and green's fractures in adults, vol 7. Lippincott Williams \& Wilkins (LWW), Philadelphia

5. Doht S, Lehnert T, Frey S, Fehske K, Jansen H, Blunk T, Meffert RH (2012) Effective combination of bone substitute and screws in the jail technique: a biomechanical study of tibial depression fractures. Int Orthop 36(10):2121-2125. doi:10.1007/s00264012-1604-8

6. Zhai Q, Luo C, Zhu Y, Yao L, Hu C, Zeng B, Zhang C (2013) Morphological characteristics of split-depression fractures of the lateral tibial plateau (Schatzker type II): a computer-tomographybased study. Int Orthop 37(5):911-917. doi:10.1007/s00264-0131825-5

7. Cross WW 3rd, Levy BA, Morgan JA, Armitage BM, Cole PA (2013) Periarticular raft constructs and fracture stability in splitdepression tibial plateau fractures. Injury 44(6):796-801. doi:10. 1016/j.injury.2012.12.028

8. Papagelopoulos PJ, Partsinevelos AA, Themistocleous GS, Mavrogenis AF, Korres DS, Soucacos PN (2006) Complications after tibia plateau fracture surgery. Injury 37(6):475-484 Epub 2005 Aug 22

9. Karunakar MA, Egol KA, Peindl R, Harrow ME, Bosse MJ, Kellam JF (2002) Split depression tibial plateau fractures: a biomechanical study. J Orthop Trauma 16(3):172-177
10. Musahl V, Tarkin I, Kobbe P, Tzioupis C, Siska PA, Pape HC (2009) New trends and techniques in open reduction and internal fixation of fractures of the tibial plateau. J Bone Joint Surg Br 91(4):426. doi:10.1302/0301-620X.91B4.20966

11. Veitch SW, Stroud RM, Toms AD (2010) Compaction bone grafting in tibial plateau fracture fixation. J Trauma 68(4):980-983. doi:10.1097/TA.0b013e3181b16e3d

12. Younger EM, Chapman MW (1989) Morbidity at bone graft donor sites. J Orthop Trauma 3(3):192-195

13. Berkes MB, Little MT, Schottel PC, Pardee NC, Zuiderbaan A, Lazaro LE, Helfet DL, Lorich DG (2014) Outcomes of Schatzker II tibial plateau fracture open reduction internal fixation using structural bone grafts. J Orthop Trauma 28(2):97-102. doi:10. 1097/BOT.0b013e31829aaee1

14. Thomas Ch, Athanasiov A, Wullschleger M, Schuetz M (2009) Current concepts in tibial plateau fractures. Acta Chir Orthop Traumatol Cech 76(5):363-373

15. Ong JC, Kennedy MT, Mitra A, Harty JA (2012) Fixation of tibial plateau fractures with synthetic bone graft versus natural bone graft: a comparison study. Ir J Med Sci 181(2):247-252. doi:10.1007/s11845-011-0797-y Epub 2012 Jan 8

16. Lubowitz JH, Vance KJ, Ayala M, Guttmann D, Reid JB 3rd (2006) Interference screw technique for arthroscopic reduction and internal fixation of compression fractures of the tibial plateau. Arthroscopy 22(12):1359

17. Blakey CM, Rennison M, Guy SP, Sutton PM (2013) A biomechanical study comparing two fixation methods in depression fractures of the lateral tibial plateau in porcine bone. BMC Sports Sci Med Rehabil 5(1):15. doi:10.1186/2052-1847-5-15

18. Pizanis A, Garcia P, Pohlemann T, Burkhardt M (2012) Balloon tibioplasty: a useful tool for reduction of tibial plateau depression fractures. J Orthop Trauma 26(7):e88-e93. doi:10.1097/BOT. 0b013e31823a8dc8

19. Wiesel SW (2010) Operative Techniques in Orthopaedic Surgery, vol 1. Lippincott Williams \& Wilkins (LWW), Philadelphia

20. Ringus VM, Lemley FR, Hubbard DF, Wearden S, Jones DL (2010) Lateral tibial plateau fracture depression as a predictor of lateral meniscus pathology. Orthopedics 33(2):80-84. doi:10. 3928/01477447-20100104-05 